\title{
The effect of poor compliance on the pharmacokinetics of carbamazepine and its epoxide metabolite using Monte Carlo simulation
}

\author{
Jun-jie DING ${ }^{1}$, Yun-jian ZHANG ${ }^{1}$, Zheng JIAO ${ }^{2, *}$, Yi WANG ${ }^{1, *}$ \\ ${ }^{1}$ Children's Hospital of Fudan University, Shanghai 201102, China; ${ }^{2}$ Department of Pharmacy, Huashan Hospital of Fudan University, \\ Shanghai 200040, China
}

\begin{abstract}
Aim: To study the effects of delayed and missed doses (poor compliance) on the pharmacokinetics of carbamazepine (CBZ) and its main active metabolite carbamazepine-10,11-epoxide (CBZE) in Chinese epilepsy patients using Monte Carlo simulation.

Methods: CBZ and CBZE time-concentration profiles in various scenarios were generated based on a population pharmacokinetic study in Chinese epilepsy patients using Monte Carlo simulation. The scenarios included patients given multiple doses of CBZ that ranged from 100 to $300 \mathrm{mg}$ three times daily or from 200 to $300 \mathrm{mg}$ every $12 \mathrm{~h}$. The therapeutic range of CBZ and CBZE for each scenario was estimated to assess the effect of delayed or missed doses and to design corresponding rescue regimens. Moreover, the impact of body weight, absorption rate and co-therapy with other antiepileptic drugs (phenytoin, phenobarbital and valproic acid) on the dosage recommendation was investigated in the event of poor compliance.

Results: The risk for a sub-therapeutic range of CBZ and CBZE was increased in a dose-dependent manner in both two and three times daily regimens when delayed or missed doses occurred. The effects of poor compliance was less prominent on the lower daily doses compared with those on the higher daily doses. The dose recommendations, in the event of poor compliance, were time related and dose dependent. Patient body weight, absorption rate and co-therapy with phenytoin, phenobarbital and valproic acid had no significant impact on the dose recommendation.

Conclusion: Patients with epilepsy should take the delayed doses as soon as they remember, and partial missed doses may need to be taken near or at the next scheduled time.
\end{abstract}

Keywords: carbamazepine; carbamazepine-10,11-epoxide; compliance; Monte Carlo simulations; nonlinear mixed effect model; population pharmacokinetics; therapeutic range

Acta Pharmacologica Sinica (2012) 33: 1431-1440; doi: 10.1038/aps.2012.135; published online 29 Oct 2012

\section{Introduction}

Carbamazepine (CBZ) is the first-line antiepileptic drug used in the treatment of complex partial (psychomotor or temporal lobe) seizures, as well as generalized tonic-clonic seizures. It is also used in combination with other antiepileptic drugs (AEDs) in patients with multiple seizure types ${ }^{[1]}$.

In humans, the most important route for CBZ metabolism is its epoxidation at the 10,11-position to the active metabolite, carbamazepine-10,11-epoxide (CBZE), which has similar antiepileptic properties to $\mathrm{CBZ}^{[2]}$. $\mathrm{CBZE}$ is thought to be partially responsible for the toxicity of CBZ treatment ${ }^{[3]}$. The poor rela-

\footnotetext{
* To whom correspondence should be addressed.

E-mail zjiao@fudan.edu.cn (Zheng JIAO). yiwang@shmu.edu.cn (Yi WANG).

Received 2012-02-06 Accepted 2012-08-26
}

tionship between dose and blood levels and the high intraand inter-individual variability of those levels were observed in CBZ therapy ${ }^{[1,4,5]}$. In addition, CBZ treatment can be further complicated by concomitant use of other AEDs with induction and inhibition properties, which may have an impact on the pharmacokinetic profiles of both CBZ and $\mathrm{CBZE}^{[6]}$. All these variations in CBZ pharmacokinetic characteristics make it necessary to individualize the dosing regimen. Maintaining plasma (or seru $\mathrm{m}$ ) concentrations within the desired therapeutic concentration is the critical issue for retaining efficacy and avoiding adverse drug effects ${ }^{[7]}$.

Epilepsy requires long-term and even life-long AEDs treatment. Adherence to the regimen is an important issue in the control of seizures ${ }^{[8-10]}$. However, delayed or missed doses occur often in the treatment of epileptic patients. It has been reported that more than $70 \%$ of respondents in one patient 
survey reported AED dose omissions ${ }^{[11]}$. A survey of insurance claims databases shows that approximately $30 \%-50 \%$ of patients with epilepsy are non-adherent to their prescribed AED therapies ${ }^{[9,12]}$. This noncompliance can lead to sub-therapeutic drug concentrations and increase the risk of seizures. Moreover, inappropriate replacement dose(s) may produce clinical toxicity, due to increased concentrations substantially above the upper limits of the therapeutic range.

Attempting to study the effect of a delayed or missed dose in patients is impractical, as it would require prospectively identifying the patients who need to delay or discontinue CBZ therapy. In addition, formal studies in patients whose medications are intentionally delayed or interrupted for experimental purposes are difficult and unacceptable for ethical reasons. To date, the impact of delayed or missed doses, with subsequent replacement of the missed dose(s) on the perturbation in the CBZ concentration-time profile, has not been examined.

Computer simulation based on population pharmacokinetics modeling provides the most appropriate means to investigate the influence of delayed or missed doses ${ }^{[13]}$. This study aimed to investigate the effect of delayed or missed CBZ doses on the pharmacokinetics of CBZ and CBZE and provide practical recommendations for the patients and clinicians by using a Monte Carlo simulation, according to our previous population pharmacokinetic study in Chinese epilepsy patients ${ }^{[14]}$.

\section{Materials and methods}

\section{Population pharmacokinetic model}

Our previous population pharmacokinetics model of CBZ and CBZE was used to generate simulation concentration data ${ }^{[14]}$. Serum samples at steady trough state $(n=459)$ were collected prospectively from 408 compliant epilepsy patients during their routine clinical care. CBZ and CBZE concentrations were simultaneously determined by high performance liquid chromatography (HPLC) ${ }^{[15]}$. Nonlinear mixed effect modeling software (NONMEM, version V level 1.1, double precision, NONMEM Project Group, San Francisco, CA, USA) was used to build the population pharmacokinetics model. The one compartment model with first order absorption and elimination was selected as the pharmacokinetic model for both CBZ and CBZE. The absorption constant rate $\left(K_{\mathrm{a}}\right)$ of $\mathrm{CBZ}$ was fixed to a literature value of $1.2 / \mathrm{h}^{[16]}$, and the transformation rate from $C B Z$ to $C B Z E$ was assumed to be equal to the elimination rate of $\mathrm{CBZ}$. The transformation ratio from $\mathrm{CBZ}$ to CBZE was fixed at 0.65 , according to the literature value ${ }^{[4]}$.

The final population pharmacokinetics model for CBZ was as follows:

$$
\begin{aligned}
\mathrm{CL} / \mathrm{F}(\mathrm{L} / \mathrm{h})=0.141 \times \text { Dose }(\mathrm{mg} / \mathrm{d})^{0.406} \times \mathrm{BW}(\mathrm{kg})^{0.117} \times \mathrm{e}^{\mathrm{\eta} 1} & \\
& \times 1.23(\text { if taking VPA and VPA daily dose }>18 \mathrm{mg} / \mathrm{kg}) \\
& \times 1.44(\text { if taking phenytoin) } \\
& \times 1.26(\text { if taking phenobarbital); } \\
\mathrm{V} / \mathrm{F}(\mathrm{L}) & =72.0 \times \mathrm{e}^{\mathrm{\eta} 2}
\end{aligned}
$$

where CL/F represents apparent clearance, V/F represents apparent volume of distribution, BW represents body weight, and VPA represents valproic acid. The between-subject variabilities (BSV) of CL/F $\left(\omega_{\mathrm{CL}}\right)$ and $\mathrm{V} / \mathrm{F}\left(\omega_{\mathrm{V}}\right)$ were $10.3 \%$ and $42.9 \%$, respectively. The proportional $\left(\sigma_{1}\right)$ and additive residual variabilities $\left(\sigma_{2}\right)$ were $14.5 \%$ and $0.67 \mathrm{mg} / \mathrm{L}$, respectively.

The final population pharmacokinetics model for CBZE was as follows:

$$
\begin{aligned}
\mathrm{CL} / \mathrm{F}(\mathrm{L} / \mathrm{h})=0.914 & \times \operatorname{Dose}(\mathrm{mg} / \mathrm{d})^{0.288} \times \mathrm{BW}(\mathrm{kg})^{0.371} \times \mathrm{e}^{\eta 1} \\
& \times 0.687(\text { if taking VPA }) ;
\end{aligned}
$$

$\mathrm{V} / \mathrm{F}(\mathrm{L})=20.5$

where the BSV of CL $\left(\omega_{\mathrm{CL}}\right)$ was $26.7 \%$. The proportional residual variability $(\sigma)$ was $26.1 \%$.

\section{Scenarios}

The scenarios were based on a 60-kg epilepsy patient who was administered multiple doses of CBZ at steady state. The patient was assumed to have complete seizure control without undesired effects. The simulation scenarios are listed below and shown in Figure 1.

CBZ (three dose levels: 100, 200, and $300 \mathrm{mg}$ ) was administered TID (7:00, 13:00, and 21:00) as a mono-therapy. (1) The

A

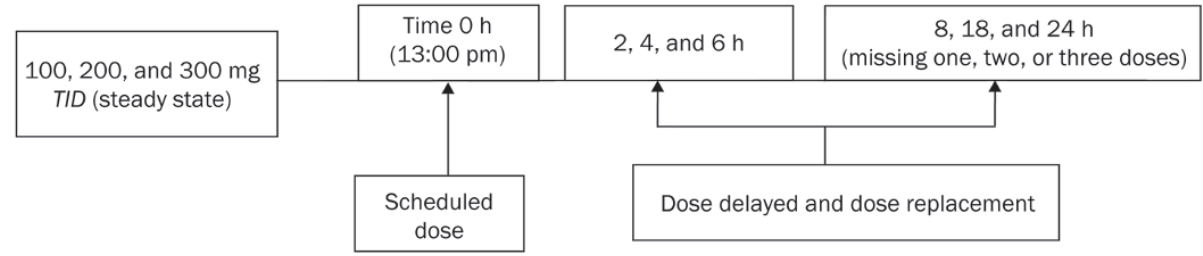

B

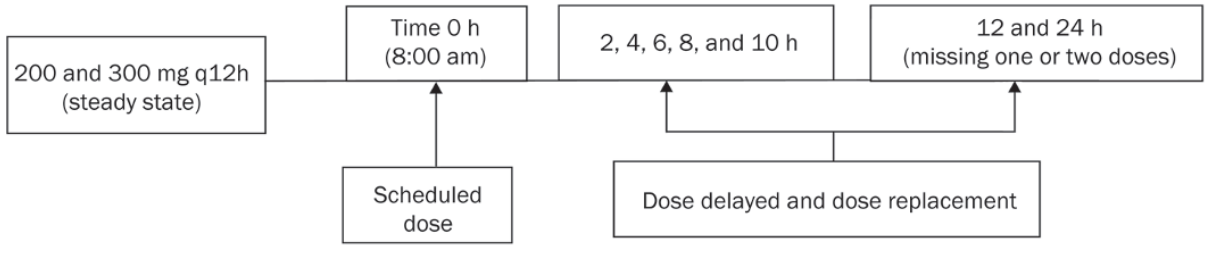

Figure 1. Simulation scenarios for delayed or missed doses on carbamazepine (CBZ) three times daily (TID, 7:00, 13:00, and 21:00) and every 12 h (q12h) regimens. (A) CBZ 100, 200, and $300 \mathrm{mg}$ TID regimens; (B) 200 and $300 \mathrm{mg}$ q12 h regimens. 
CBZ dose at noon (13:00) was taken $2,4,6,8,18$, or $24 \mathrm{~h}$ after the scheduled time. (2) The missed dose or partial missed dose of CBZ was taken at the next scheduled time, 21:00 or 7:00 (ie, as the replacement dose for the missed dose, Figure $1 \mathrm{~A})$.

CBZ (two doses levels: 200 and $300 \mathrm{mg}$ ) was administered q12 $\mathrm{h}$ as a mono-therapy. (1) The missed dose of CBZ was taken at 2, 4, 6, 8, 10, 12, or $24 \mathrm{~h}$ after the scheduled time. (2) The missed dose or partial missed dose of CBZ was taken at the next scheduled time (ie, the replacement dose for the missed dose, Figure 1B).

\section{Simulation}

Monte Carlo simulations with nested random effects were conducted using the \$SIMULATION block in the NONMEM software (Version 7.2; Icon Inc, PA, USA) with the ONLYSIMULATION and SUBPROBLEMS option. Five thousand virtual patients, with time-concentration profiles of both $C B Z$ and CBZE, were generated using the CBZ and CBZE population model parameters (eg, pharmacokinetic parameters, BSV and residual variability) previously described for each scenario. The summary of pharmacokinetic parameters of CBZ and CBZE employed in the simulation are listed in Table 1.

Table 1. The summary of apparent clearance (CL) and apparent distribution (V) values for carbamazepine (CBZ) and carbamazepine10,11-epoxide (CBZE) employed in the simulation.

\begin{tabular}{lclc}
\hline & $\begin{array}{c}\text { CL for CBZ } \\
(\mathrm{L} / \mathrm{h})\end{array}$ & \multicolumn{1}{c}{$\begin{array}{c}\mathrm{V} \text { for } \mathrm{CBZ} \\
(\mathrm{L})\end{array}$} & $\begin{array}{c}\mathrm{CL} \text { for CBZE } \\
(\mathrm{L} / \mathrm{h})\end{array}$ \\
\hline Geometric mean (SD) & $3.05(0.31)$ & $72.7(37.5)$ & $26.42(7.17)$ \\
Median & 3.06 & 72.6 & 26.24 \\
$90 \% \mathrm{Cl}$ & $2.57-3.60$ & $36.1-145.1$ & $16.84-41.11$ \\
$99 \% \mathrm{Cl}$ & $2.32-3.95$ & $24.6-215.7$ & $12.68-50.92$ \\
Range & $2.08-4.49$ & $18.1-372.1$ & $8.78-71.31$ \\
\hline
\end{tabular}

Cl: confidence internal.

\section{Summary of simulations' outcomes}

The therapeutic range of CBZ and CBZE for each scenario was defined as the 5th-95th percentile of the simulated data and was employed in the further analysis, according to the most recent guidelines in therapeutic drug monitoring of AEDs $^{[1,17-19]}$.

The outcomes were summarized as follows:

(1) Percentages of individuals with $C_{\min }$, which was defined as the minimum concentration below the lower limit of the therapeutic range (5th percentile of simulated $C_{\text {min }}$ ) of $\mathrm{CBZ}$ and CBZE after taking the last dose and the replaced doses;

(2) Percentages of individuals with $C_{\max }$, which was defined as the maximum concentration above the upper limit of the therapeutic range (95th percentile of simulated $C_{\max }$ ) of $\mathrm{CBZ}$ and CBZE after the replacement dose and next scheduled dose was taken, which indicates the probability of potential toxicity;

(3) The percentage difference beyond the therapeutic range between full compliance and taking replaced doses after a delayed or missed dose.

Post processing of output was undertaken by the R package (version 2.12.1, www.r-project.com).

\section{Sensitivity analysis}

When a sequence of mono-therapies with AEDs is unsuccessful, combination therapy is usually tried in an attempt to improve efficacy, tolerability or both ${ }^{[20]}$. A combination of phenytoin (PHT), phenobarbital (PB), valproic acid (VPA) and CBZ is the most commonly used combination therapy in the management of refractory epilepsy ${ }^{[18,19]}$. In addition, the previous population pharmacokinetics study showed that a patient's weight has a significant impact on the CL for both CBZ and CBZE ${ }^{[14]}$. Moreover, the $K_{\mathrm{a}}$ in the population pharmacokinetics model of CBZ was fixed to $1.2 / \mathrm{h}$ and its BSV was set to zero, which may not represent the real clinical setting. Therefore, it is very helpful to investigate the influence of the patient's weight, $K_{\mathrm{a}}$ and the combination with other AEDs on the dosage recommendation in the event of poor compliance. The scenarios in which patients with 40or $80-\mathrm{kg}$ of body weight, $30 \%$ or $50 \%$ of $\omega_{\mathrm{ka}}, 0.6$ or $1.8 / \mathrm{h}$ of $K_{\mathrm{a}}$ and combination with other AEDs were chosen to perform the sensitivity analysis.

Because the fixed dosing interval (7:00, 13:00, and 21:00, ie, dosing interval of $6 \mathrm{~h}-8 \mathrm{~h}-10 \mathrm{~h}$ ) in the current study may not reflect the real clinical practice, the effect on the dose recommendation of various dosing intervals, such TID (from 8-8-8 h to $4-8-12 \mathrm{~h}$ ) and BID (from 10-14 h to 14-10 h) regimens, were also investigated (Table 2).

Table 2. The various dosing intervals employed in the sensitivity analysis.

\begin{tabular}{ll}
\hline \multicolumn{1}{c}{ Dosing intervals } & \multicolumn{1}{c}{ Dosing time } \\
\hline $6,8,10 \mathrm{~h}$ (reference) & $7: 00,13: 00,21: 00$ \\
$6,6,12 \mathrm{~h}$ & $8: 00,14: 00,20: 00$ \\
$6,10,8 \mathrm{~h}$ & $6: 00,12: 00,22: 00$ \\
$8,8,8 \mathrm{~h}$ & $6: 00,14: 00,22: 00$ \\
$8,6,10 \mathrm{~h}$ & $6: 00,14: 00,20: 00$ \\
$4,8,12 \mathrm{~h}$ & $8: 00,12: 00,20: 00$ \\
$4,10,10 \mathrm{~h}$ & $8: 00,12: 00,22: 00$ \\
$12,12 \mathrm{~h}$ (reference) & $8: 00,20: 00$ \\
$14,10 \mathrm{~h}$ & $7: 00,21: 00$ \\
$10,14 \mathrm{~h}$ & $9: 00,19: 00$ \\
\hline
\end{tabular}

Notes: Three times daily (TID) doses were given at 6:00-8:00,

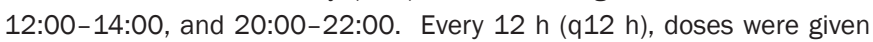
at 7:00-9:00 and 19:00-21:00. The opposite boundaries of the dosing windows were used to perform the sensitivity analysis.

Furthermore, in real clinical settings, a time window of 30 min for a scheduled dose intake is a typical situation for many epileptic patients. Therefore, this "noise" was added to the simulation scenario as a sensitivity analysis to investigate its impact on the time-concentration profile of CBZ and CBZE. 


\section{Results}

\section{Summary of simulation results}

The individual clearance of CBZ and CBZE used in the simulation ranged from 2.21 to $4.15 \mathrm{~L} / \mathrm{h}$ (median $3.06 \mathrm{~L} / \mathrm{h}$ ) and 8.78 to $71.31 \mathrm{~L} / \mathrm{h}$ (median $26.24 \mathrm{~L} / \mathrm{h}$ ), respectively. The patients with slower CBZ and CBZE clearance contributed to higher time-concentration profiles.

Figure 2 shows that the percentages of subjects that had subtherapeutic ranges of both CBZ and CBZE were dose dependent and time related. The risk of having a sub-therapeutic range of CBZ was increased from $5.0 \%$ (full compliance) to $80.2 \%, 93.1 \%$, and $95.7 \%$ in terms of scheduled dosing delayed up to $24 \mathrm{~h}$ from the scheduled time for CBZ $100 \mathrm{mg}$ TID, 200 $\mathrm{mg}$ TID, and $300 \mathrm{mg}$ TID mono-therapy, respectively (Figure 2A). Regarding a regimen of CBZ $200 \mathrm{mg} \mathrm{q12} \mathrm{h} \mathrm{and} 300 \mathrm{mg}$ $\mathrm{q} 12 \mathrm{~h}$, the risk of sub-therapeutic ranges of CBZ was increased from $5.0 \%$ to $80.3 \%$ and $86.7 \%$ when scheduled dosing was delayed up to $24 \mathrm{~h}$, respectively. The percentages of subtherapeutic ranges of CBZE show the same trend with CBZ but were lower than those of CBZ (Figure 2B).
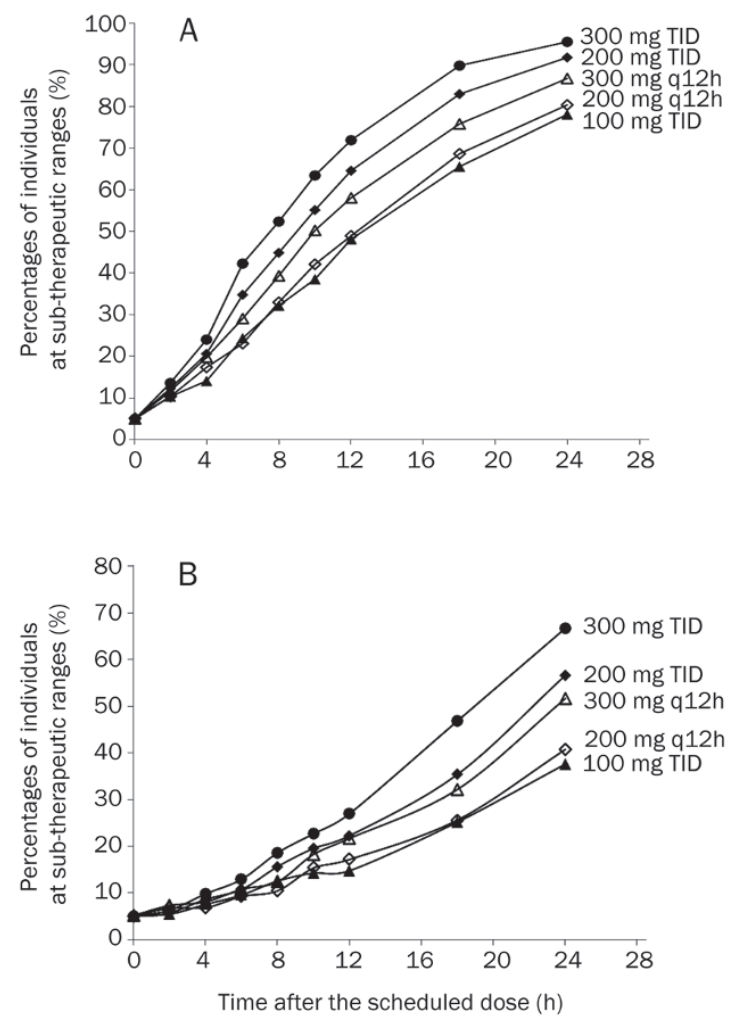

Figure 2. The percentage of the sub-therapeutic range of carbamazepine (CBZ) and carbamazepine-10,11-epoxide (CBZE) concentrations with different times after the last dose (13:00) in CBZ mono-therapy of three times daily regimens (TID, 7:00, 13:00, and 21:00) and every $12 \mathrm{~h}$ regimens (q12h). (A) CBZ; (B) CBZE.

The differences in the percentage of outside the therapeutic range of CBZ and CBZE between full compliance and taking a replaced dose after a delayed or missed dose, in all scenarios, are shown in Figure 3. If a patient waits to take the delayed dose until the next scheduled time, the difference in percentage of drug concentrations above the upper limit of the CBZ therapeutic range, between the concentrations after full compliance and the concentrations in the next dosing interval after taking a replaced dose, was increased. For example, when a patient takes a regimen of $200 \mathrm{mg}$ TID, the difference in percentage of concentrations beyond the upper limit of the therapeutic range was 5.9\% with taking the delayed dose $(200 \mathrm{mg}) 6$ $\mathrm{h}$ later, compared to that of $2.5 \%$ with taking the delayed dose $2 \mathrm{~h}$ later (Figure 3A).

If one, two or three consecutive doses were missed, the inappropriate replaced dose can increase the risk of $\mathrm{CBZ}$ deviation from the therapeutic range (Figure 3). For example, regarding CBZ $200 \mathrm{mg}$ TID regimens, the difference of the percentage of sub-therapeutic range between full compliance and taking a $200 \mathrm{mg}$ replaced dose $8 \mathrm{~h}$ after non-compliance (the next scheduled time) was $12.2 \%$, in the case of one missed dose, while the difference related to beyond the upper limit of the therapeutic range was $10.3 \%$ after taking a $500 \mathrm{mg}$ replaced dose, in the case of two missed doses (Figure 3A).

The results also show that in poorly compliant patients, the impact of a replaced dose on the CBZE concentration profile was lower than that on the CBZ concentration profile (Figure $3)$. In most of the scenarios, the trends of deviation from the therapeutic range of CBZE, after taking a replaced dose, were consistent with those from the therapeutic range of CBZ. Inconsistency was only observed after taking a replaced dose when 2 or 3 consecutive doses were missed (Figure 3). For example, in the CBZ $200 \mathrm{mg}$ TID regimen with 2 doses missing scenario, after taking a $500 \mathrm{mg}$ replacement dose at the next scheduled time, the concentration profile of CBZE was closer to the therapeutic range of CBZE. However, the concentration profile of $\mathrm{CBZ}$ deviated further from the therapeutic range (Figure 3A).

\section{Sensitivity analysis}

Two scenarios of replacement doses at the next scheduled time after missing one and two consecutive doses for CBZ TID regimens were chosen to perform the sensitivity analysis. The analysis results were presented in Figure 4. The results show that body weight of the patient, $K_{\mathrm{a}}$ and co-therapy with phenytoin, phenobarbital and valproic acid had no significant impact on the dose recommendation in poorly compliant patients. In the percent of outside the therapeutic ranges of both $\mathrm{CBZ}$ and CBZE, most of the differences between full compliance and poor compliance, while followed by taking a replaced dose, were less than $5 \%$, as well as almost equal to that of a patient of $60 \mathrm{~kg}$ with CBZ monotherapy. Only a slight deviation of the upper limit of the therapeutic range of both CBZ and CBZE was observed in the case of co-therapy with phenytoin, phenobarbital or valproic acid. A dose taken $30 \mathrm{~min}$ before or delayed from the scheduled time has no significant impact on the time-concentration profile of both CBZ and CBZE.

The impact of dosing intervals on the dose recommendation 

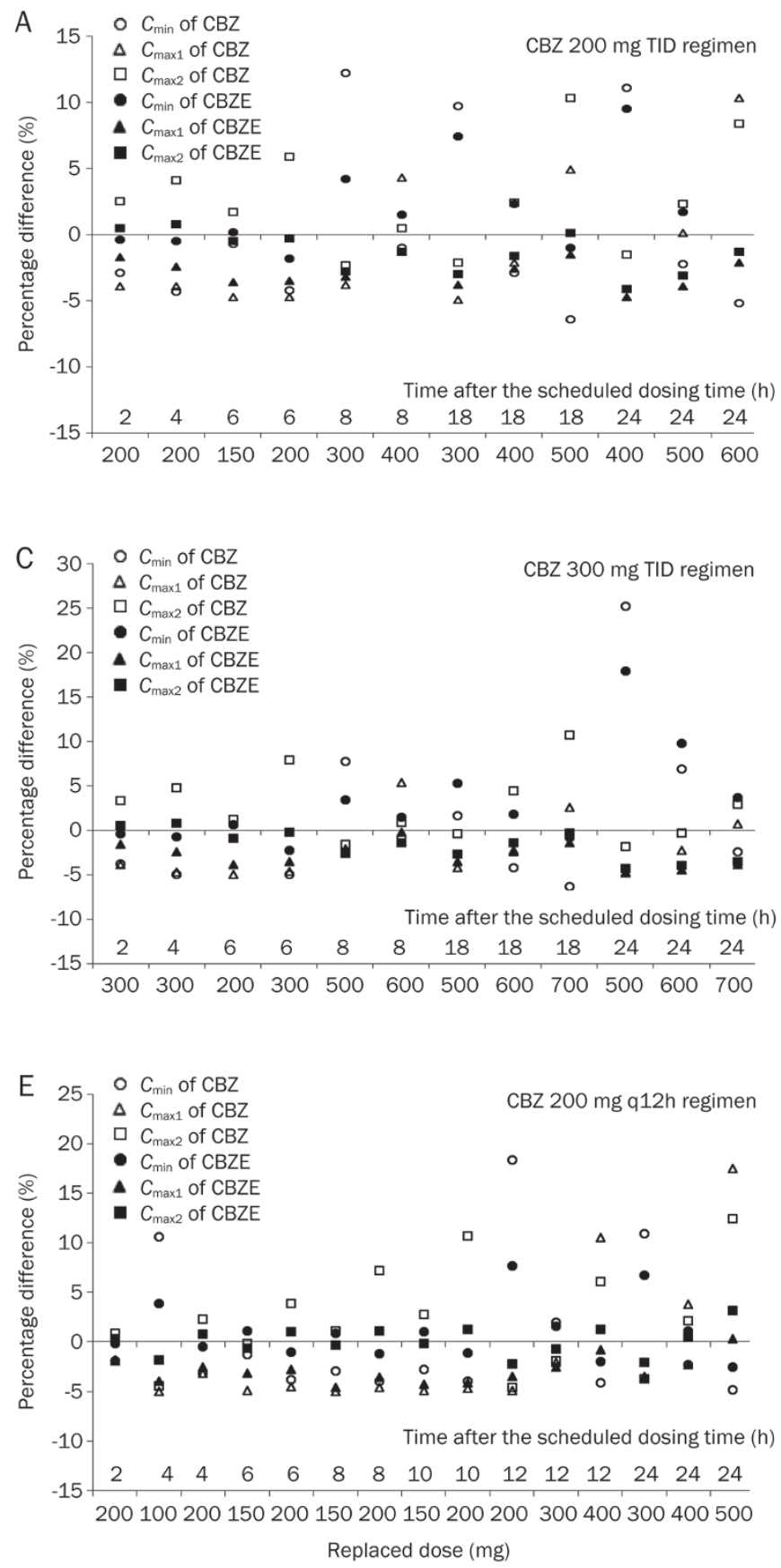

was shown in Figure 5. The time-concentration profiles of CBZ followed by taking a replaced dose in 8 selected dosing intervals ( 6 for TID regimen, 2 for BID regimen) deviated slightly from referral dosing intervals $(6-8-10 \mathrm{~h}$ for TID regimen, $\mathrm{q} 12 \mathrm{~h}$ for BID regimen). The differences in the percent of the outside the therapeutic range of both CBZ and CBZE between full compliance and selected dosing regimen were less than $6 \%$.

\section{Dosing recommendations}

Based on the above analysis, the resumed dosing recommendations for delayed and missed doses of CBZ were shown in
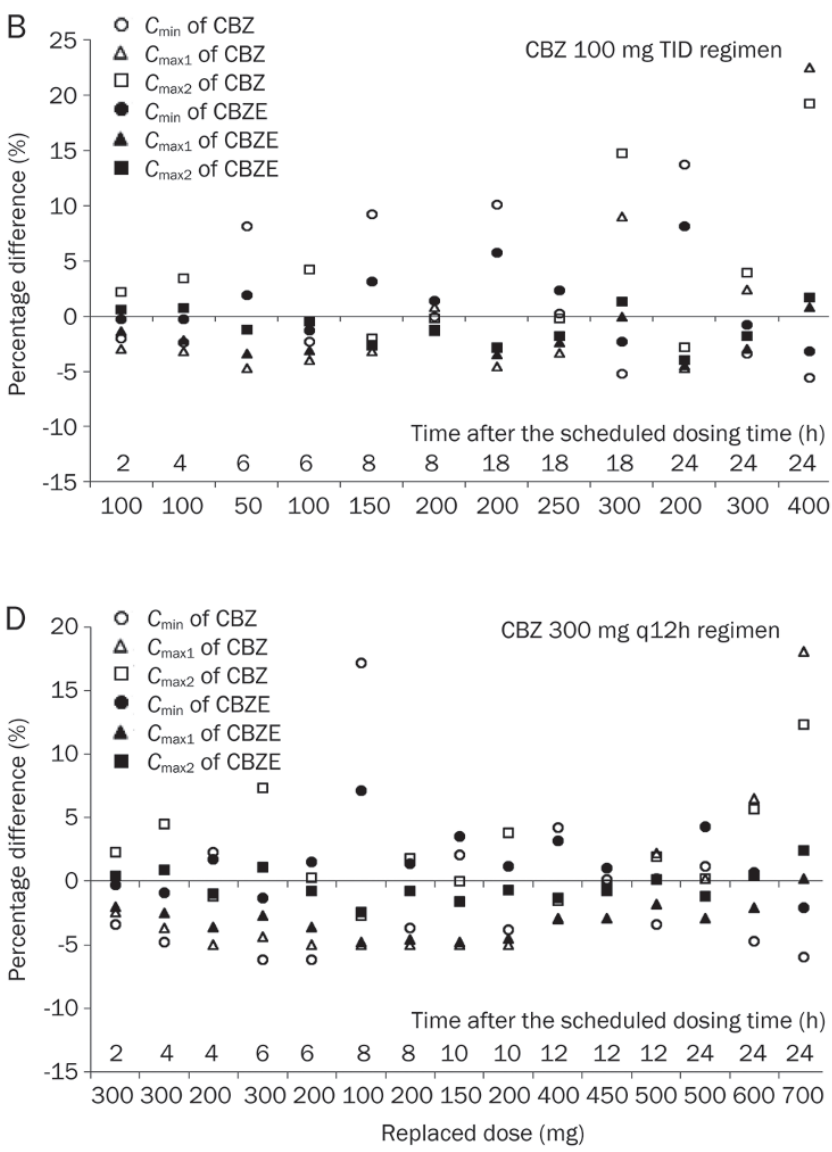

Figure 3. The difference in the percentage of concentrations outside the therapeutic range of carbamazepine (CBZ) and carbamazepine-10,11epoxide (CBZE) between complete compliance and poor compliance, followed by the results of administering a replacement dose. (A) CBZ $200 \mathrm{mg}$ three times daily (TID, 7:00, 13:00 and 21:00) regimen; (B) CBZ $100 \mathrm{mg}$ TID regimen; (C) CBZ $300 \mathrm{mg}$ TID regimen; (D) CBZ $300 \mathrm{mg}$ every $12 \mathrm{~h}$ ( $\mathrm{q} 12 \mathrm{~h}$ ) regimen; (E) CBZ $200 \mathrm{mg}$ q12h regimen. $C_{\min }$ : minimum concentration after taking the replaced dose; $C_{\max 1}$ : maximum concentration after taking replacement dose; $C_{\max 2}$ : maximum concentration after taking the next scheduled dose.

Table 3, Figure 6, and Figure S1. The patients should take the delayed dose upon remembering a missed dose that had been delayed up to $4 \mathrm{~h}$ for the CBZ 100-300 $\mathrm{mg}$ TID and 200-300 $\mathrm{mg} \mathrm{q} 12 \mathrm{~h}$ regimens. For a delay of more than $4 \mathrm{~h}$ until the next scheduled time, a partial scheduled dose was recommended, except in the CBZ $100 \mathrm{mg}$ TID regimens.

If one or two consecutive doses were missed, replacement of double doses at the next scheduled time are recommended for CBZ 100 or $200 \mathrm{mg}$ TID regimens (Figure S1A1-A4 and Figure 6A1-A4), and $500 \mathrm{mg}$ replaced doses are suggested for $300 \mathrm{mg}$ TID regimens (Figure S1B1-B4). If three doses were missed, 300,400 , and $500 \mathrm{mg}$ replaced doses are suggested in terms of 

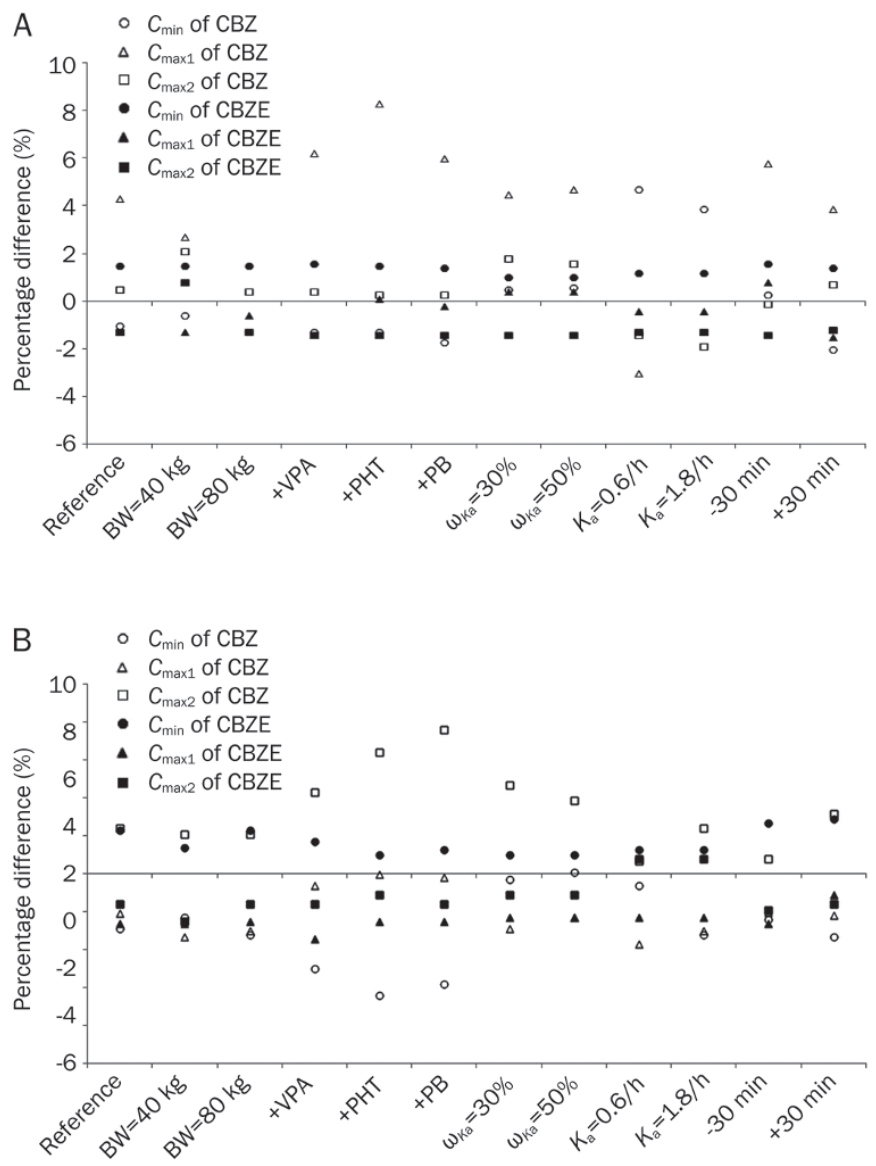

Figure 4. The impact of body weight (BW), absorption rate $\left(K_{\mathrm{a}}\right)$ and cotherapy with other antiepileptic drugs on the dosage recommendation in the event of poor compliance. (A) CBZ $200 \mathrm{mg}$ three times daily (TID, 7:00, 13:00, and 21:00) regimens, one dose (200 $\mathrm{mg}$ ) was missed and double doses $(400 \mathrm{mg}$ ) were taken at the next scheduled time. (B) CBZ $200 \mathrm{mg}$ TID regimens, two consecutive doses (400 mg) were missed and double doses (400 $\mathrm{mg}$ ) were taken at the next scheduled time. CBZ: carbamazepine; CBZE: carbamazepine-10,11-epoxide; $C_{\min }$ : minimum concentration after taking the replaced dose; $C_{\max 1}$ : maximum concentration after replacement dose; $C_{\max 2}$ : maximum concentration after taking the next scheduled dose. BW: body weight; +VPA: co-therapy with valproic acid; +PHT: co-therapy with phenytoin; +PB: co-therapy with phenobarbital; $\omega_{\text {Ka: }}$ : between subject variability of $K_{\mathrm{a}}$; $-30 \mathrm{~min}$ : taking dose 30 min before scheduled dose; +30 min: taking dose 30 min after scheduled dose. Reference: the model was based on a 60-kg compliant epilepsy patient whose $K_{\mathrm{a}}$ of CBZ was $1.2 / \mathrm{h}$ and $\omega_{\text {Ka }}$ was fixed to zero.

CBZ 100, 200, and $300 \mathrm{mg}$ TID regimens, respectively (Figure S1A5-A6, Figure 6A5-A6, and Figure S1B5-B6). If one or two doses were missed, a replacement of 300 or $400 \mathrm{mg}$ is recommended for CBZ $200 \mathrm{mg}$ q12h regimens, respectively (Figure S1C1-C4), and 450 or $500 \mathrm{mg}$ replaced doses are suggested for CBZ $300 \mathrm{mg}$ q12h regimens, respectively (Figure S1D1-D4).

Moreover, our study indicated that the same replaced doses (500 mg) could be recommended when missing a dose in regimens with the same total daily dose (eg, $200 \mathrm{mg}$ TID and 300 mg q12h regimens).
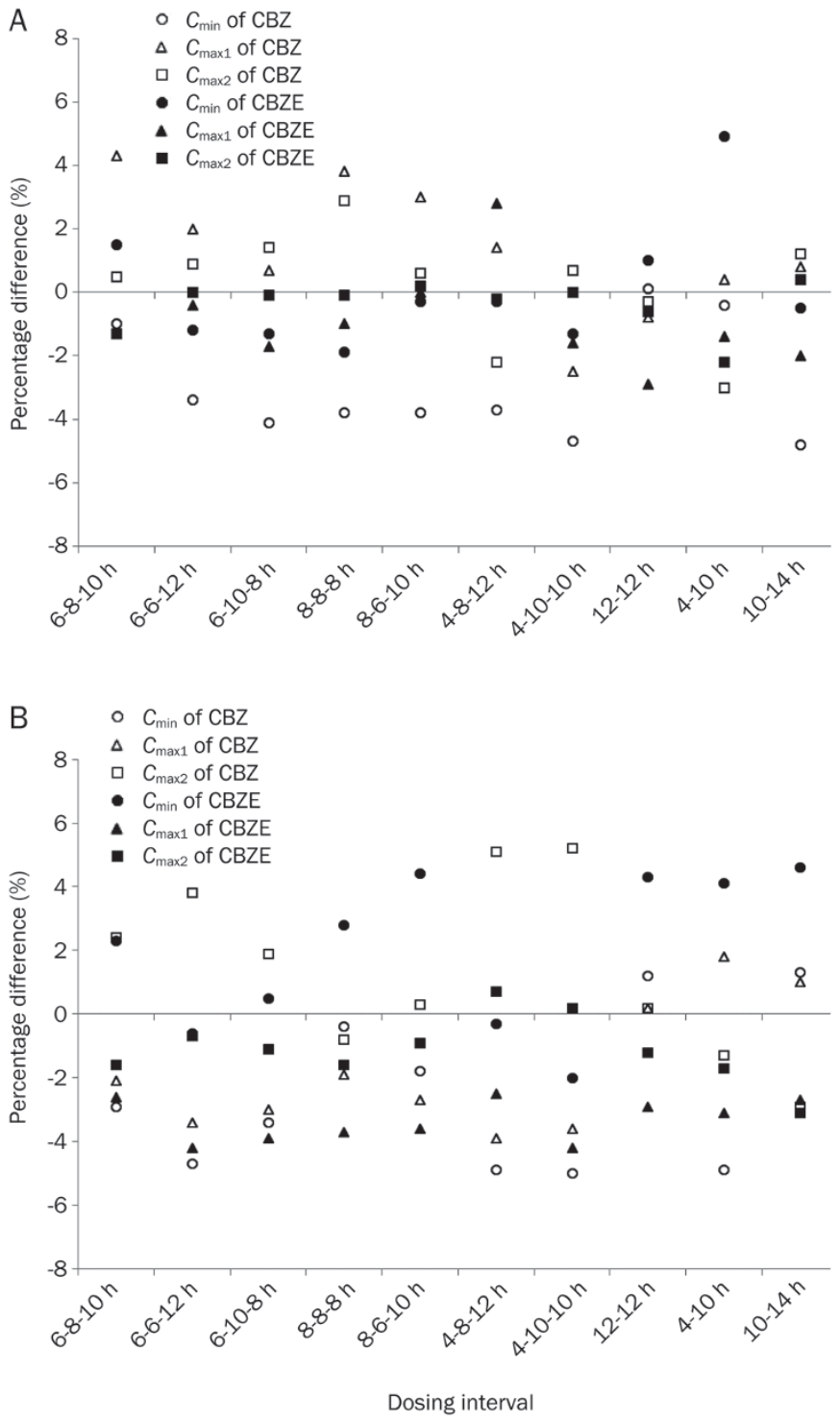

Figure 5. The impact of doing intervals on the dosage recommendation in the event of poor compliance. (A) One dose was missed, and 400 or 450 $\mathrm{mg}$ replacement doses were taken at the next scheduled time in terms of $200 \mathrm{mg}$ TID and $300 \mathrm{mg}$ q12h regimens. (B) Two consecutive doses were missed, and 400 or $500 \mathrm{mg}$ replacement doses were taken at the next scheduled time in terms of $200 \mathrm{mg}$ TID and $300 \mathrm{mg} \mathrm{q} 12 \mathrm{~h}$ regimens. Three times daily (TID) dosing regimens were taken at dosing intervals from 4-10-10 $\mathrm{h}$ to 8-8-8 $\mathrm{h}$. Two times daily (BID) dosing regimens were taken at dosing intervals of 10-14 h and 14-10 h. CBZ: carbamazepine; CBZE: carbamazepine-10,11-epoxide; $C_{\text {min }}$ : minimum concentration after taking the replaced dose; $C_{\max 1}$ : maximum concentration after taking replacement dose; $C_{\max 2}$ : maximum concentration after taking the next scheduled dose.

\section{Discussion}

To our knowledge, this is the first study to investigate the impact of poor compliance on pharmacokinetics of both CBZ and CBZE based on therapeutic range in epilepsy patients administered conventional formulations of CBZ. Garnett $e t$ 
Table 3. Dosing recommendations for epileptic patients with a 40-80 kg body weight according to delayed or missed doses on carbamazepine three times daily (TID, dosing interval of $4-8,6-10$, and $8-12 \mathrm{~h}$ ) or every $12 \mathrm{~h}$ regimens (q12h, dosing interval of $10-14 \mathrm{~h}$ ).

\begin{tabular}{|c|c|c|}
\hline Dosage & Scenarios & Dosing recommendation \\
\hline \multirow[t]{4}{*}{100 mg TID } & One dose at noon was delayed up to $6 \mathrm{~h}$ & Take 100 mg immediately \\
\hline & Missed noon one dose & Take $200 \mathrm{mg}$ at night \\
\hline & Missed two consecutive (noon and night) doses & Take $200 \mathrm{mg}$ at morning \\
\hline & Missed three consecutive doses & Take $300 \mathrm{mg}$ at the next scheduled time \\
\hline \multirow[t]{3}{*}{200 mg TID } & One dose at noon was delayed up to $4 \mathrm{~h}$ & Take 200 mg immediately \\
\hline & Missed two consecutive (noon and night) doses & Take $400 \mathrm{mg}$ at morning \\
\hline & Missed three consecutive doses & Take $500 \mathrm{mg}$ at the next scheduled time \\
\hline \multirow[t]{3}{*}{300 mg TID } & One dose at noon was delayed up to $4 \mathrm{~h}$ & Take 300 mg immediately \\
\hline & One dose at noon was delayed $4-6 \mathrm{~h}$ & Take 200 mg immediately \\
\hline & Missed noon one dose & Take $500 \mathrm{mg}$ at night \\
\hline \multirow{3}{*}{$200 \mathrm{mg}$ q12h } & One dose was delayed $8-12 \mathrm{~h}$ & Take 100 mg immediately \\
\hline & Missing one dose & Take $300 \mathrm{mg}$ at the next scheduled time \\
\hline & Missing two consecutive doses & Take $400 \mathrm{mg}$ at the next scheduled time \\
\hline \multirow[t]{5}{*}{$300 \mathrm{mg}$ q12h } & One dose was delayed up to $4 \mathrm{~h}$ & Take 300 mg immediately \\
\hline & One dose was delayed $4-8 \mathrm{~h}$ & Take 200 mg immediately \\
\hline & One dose was delayed $8-12 \mathrm{~h}$ & Take 150 mg immediately \\
\hline & Missing one dose & Take $450 \mathrm{mg}$ at the next scheduled time \\
\hline & Missing two consecutive doses & Take $500 \mathrm{mg}$ at the next scheduled time \\
\hline
\end{tabular}

$a l^{[21,22]}$ previously investigated the effect of delayed and missed doses on the concentration of the extended release capsule of CBZ. In these two studies, the pharmacokinetic parameters used for simulation were derived from 12 healthy volunteers, and the impact of covariates, such as weight, dose and comedications was not considered. In addition, the BSV and residual variability were not incorporated into the simulation, which may not represent the real clinical settings. Furthermore, the impact of poor compliance on the pharmacokinetics of its active metabolite CBZE was not investigated in these studies.

Compliance with drug treatment is an important issue in treatment for chronic diseases such as epilepsy, cardiovascular diseases and diabetes, etc. Poor compliance can increase the onset risk of these diseases. For epilepsy, compliance is critical to the control of seizures. An early study reported that $31 \%$ of seizures may be related to poor compliance ${ }^{[10]}$. Another study found that the seizure risk was $21 \%$ higher among patients with poor compliance than those with good compliance ${ }^{[8]}$. In adults with epilepsy, poor compliance has been found to be associated with increased hospital visits, as well as increased medical costs and elevated mortality ${ }^{[12,23]}$. As delayed or missed doses are inevitable in long-term therapy, it is necessary to investigate its impact on pharmacokinetics and to make rational recommendation for a rescue dosing regimen based on pharmacokinetic modeling and simulations.

Maintaining CBZ and CBZE concentration in the suitable range will determine the effectiveness of chronic therapy for patients with epilepsy. Retrospective and observational studies suggest that the reference ranges for CBZ and CBZE are 4-12 $\mathrm{mg} / \mathrm{L}$ and $0.8-3.2 \mathrm{mg} / \mathrm{L}$, respectively ${ }^{[5,17]}$. The reference range has been a controversial concept, partly because it was initially defined on the basis of limited data for individual AEDs, which may not describe adequately the concentrationresponse relationship in patients with epilepsy ${ }^{[7]}$. It may not be uncommon for patients to respond well to serum AED concentrations below the commonly reported lower limit of the reference range ${ }^{[24,25]}$. Moreover, the upper limit of the range may vary from one patient to another. Some patients may have toxic symptoms at low drug concentrations, while others may tolerate high drug concentrations ${ }^{[26]}$. In recent years, the tendency in epilepsy management is changing from reference ranges to therapeutic ranges (or individual therapeutic concentrations $)^{[7,27]}$. The latter can be defined as the concentration (or range of concentrations) that has been empirically found to produce the optimal response in the individual patient (ie, complete seizure control without undesired effects or, if that goal is not achievable, the best compromise between seizure suppression and concentration-related adverse effects). Therefore, in our study, the therapeutic range, instead of the

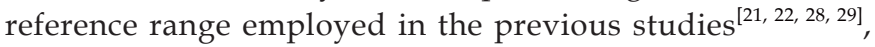
was used to assess the effect of missed or delayed doses and to make dose recommendations.

Our simulation results show that the effect of delayed or 

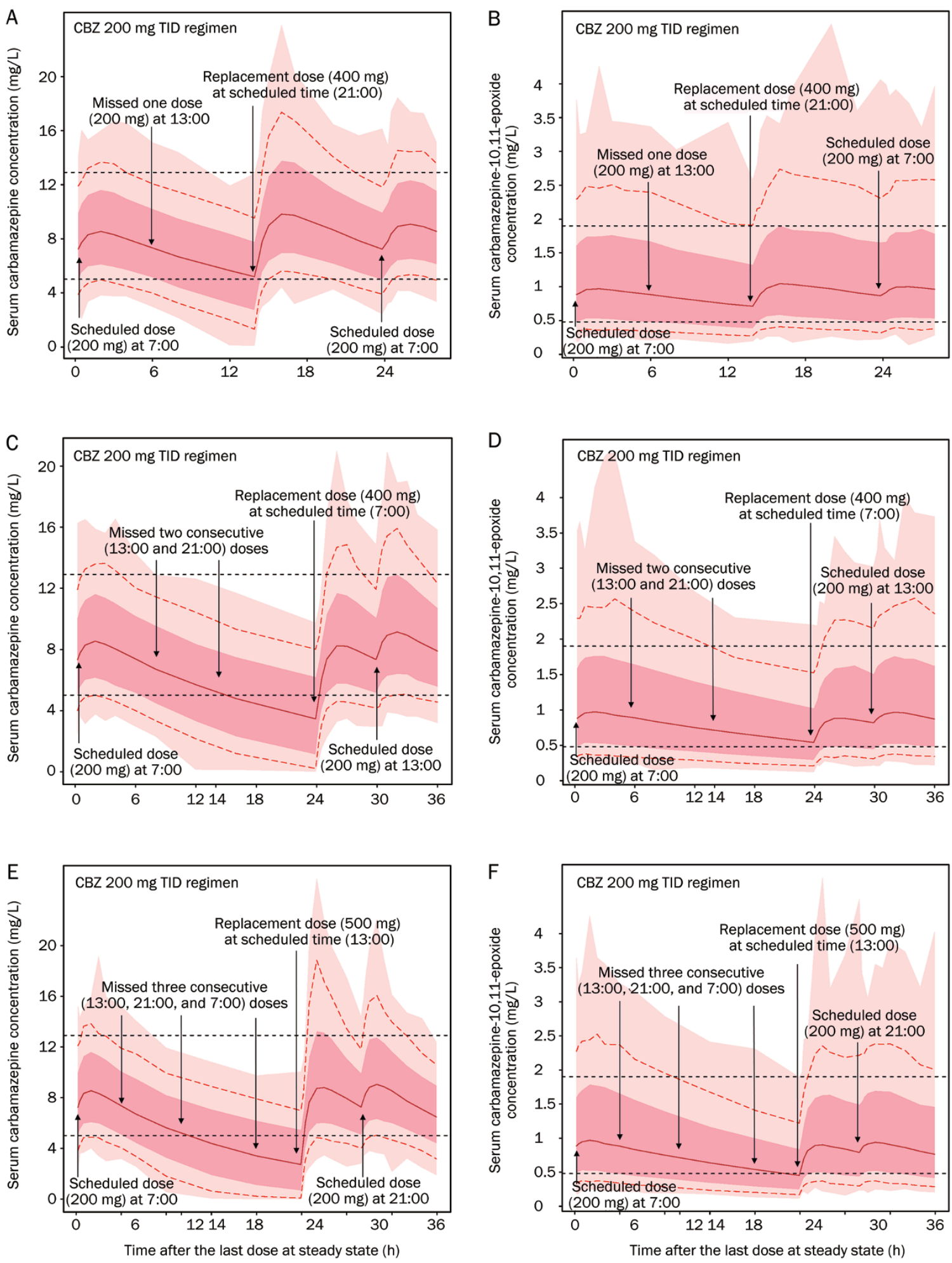

Figure 6. Carbamazepine (CBZ) and carbamazepine-10,11-epoxide (CBZE) concentration-time profiles from Monte-Carlo simulation of 5000 virtual epilepsy patients with 60-kg body weight while at steady state and after missing one, two or three scheduled doses, with recommended replacement doses followed by resumption of scheduled therapy after the replaced dose. The deep pink and the light pink colors represent the 5th-95th percentiles and outside 5th-95th percentiles of the simulated data, respectively. The red solid line represents the median, and the red dotted lines represent the 0.5th and 99.5th percentiles of the simulated data. The dotted black horizontal lines represent the lower and upper boundaries of the therapeutic range of CBZ and CBZE. (A, B) One dose (200 mg) was missed on a CBZ $200 \mathrm{mg}$. Three times daily (TID) regimen, and a $400 \mathrm{mg}$ replacement dose was taken. (C, D) Two doses (400 mg) were missed on a CBZ $200 \mathrm{mg}$ TID regimen, and a $400 \mathrm{mg}$ replacement dose was taken. (E, F) Three doses (600 $\mathrm{mg}$ ) were missed on a CBZ $200 \mathrm{mg}$ TID regimen, and a $500 \mathrm{mg}$ replacement dose was taken. 
missed doses on steady state CBZ concentrations depends on the daily dose and administration. The chance for a subtherapeutic range of CBZ and CBZE was increased in a daily dose-dependent manner in both TID and $\mathrm{q} 12 \mathrm{~h}$ regimens with delayed or missed doses. This result was largely due to the non-linear effect of daily dose on clearance. Moreover, the risks of sub-therapeutic ranges of both CBZ and CBZE from a dosing regimen of TID were slightly higher than those from a regimen of $\mathrm{q} 12 \mathrm{~h}$ of the same daily dose. This increased risk could be attributed to small fluctuations of peak-to-trough in TID regimens, which were more sensitive to the delayed dose than were the $\mathrm{q} 12 \mathrm{~h}$ regimens, according to the therapeutic range. The effect of delaying or missing two doses is more pronounced for both TID and $\mathrm{q} 12 \mathrm{~h}$ regimens, especially in high daily dose regimens. There may be considerable risk of sub-therapeutic ranges, and the two missed doses must be replaced as soon as possible.

How many doses need to be taken depends on how long the dose is delayed from the scheduled dosing time and on the dosing regimen. For example, at a dosing regimen of CBZ 200 $\mathrm{mg}$ TID, if the dose delay were brief (up to $4 \mathrm{~h}$ ), then taking one dose $(200 \mathrm{mg}$ ) could maintain an appropriate trough-peak concentration of both CBZ and CBZE. However, taking one dose $6 \mathrm{~h}$ late could increase the risk of concentrations outside the upper limit of the therapeutic range of CBZ and, therefore, a $150 \mathrm{mg}$ replaced dose is recommended. If one dose were missed in this patient, a $400 \mathrm{mg}$ replaced dose was shown to be most appropriate. However, if two or three doses were missed, 3- or 4-fold replaced doses (300 or $600 \mathrm{mg}$ ) were not needed. In such cases, 400 and $500 \mathrm{mg}$ replaced doses, respectively, were recommended. This result could be attributed to the nonlinear pharmacokinetic characteristics of CBZ and the narrow therapeutic range ${ }^{[14]}$. In most scenarios, the risk of having a concentration of CBZE, outside the therapeutic range was slightly lower than that of CBZ if replacement doses were administered (Figure 3A and Table 3).

In our study, Monte Carlo simulations, based on the previous population pharmacokinetics study, were used to examine the concentration profiles of both CBZ and CBZE in terms of poor compliance. The total daily doses chosen for the analysis, ie, c. $300-900 \mathrm{mg} / \mathrm{d}$, represent most common clinical settings ${ }^{[14,30]}$, permitting our results and interpretation of the results to be generalized for the majority of patients on CBZ therapy. Moreover, introduction of inter-patient and residual variability into the model has added to the reliability of our predictions. The most common co-therapy medications used with $\mathrm{CBZ}$ are $\mathrm{PB}, \mathrm{PHT}$, and $\mathrm{VPA}$, which have a significant impact on the clearance of CBZ and/or CBZE. Therefore, the influence of co-therapies on the pharmacokinetic profile of CBZ and CBZE was investigated. Meanwhile, sensitivity analysis was performed on the patients, who had a wide range of individual body weight and different levels of $K_{\mathrm{a}}$ which may make the results more applicable in clinical practice.

In our study, population pharmacokinetic parameters were estimated from HPLC measurement. Currently, CBZ concentration is also determined by a fluorescence polarization immunoassay (FPIA) on the TDx/TDx FLx system in some laboratories. The immunoassay is not specific to the CBZ determination and can theoretically result in overestimation because of the cross-reaction of the assay antibody with its metabolite $\mathrm{CBZE}^{[31-33]}$. The previous study reported that the measurements by FPIA were $4.58 \% \pm 6.64 \%$ higher than those obtained with HPLC ${ }^{[32]}$, which has no pronounced impact on the population pharmacokinetic analysis ${ }^{[1,31,33]}$. Therefore, the result of our study may also apply in the case of FPIA measurement.

This simulation study has several limitations. As all the concentration-time data collected in previous population pharmacokinetics studies of CBZ and CBZE were trough values, $K_{\mathrm{a}}$ and $\mathrm{V}$ cannot be accurately estimated. Therefore, $K_{\mathrm{a}}$ of CBZ and V of CBZE were fixed in this study, which may have an impact on the simulated peak concentration of CBZ and CBZE. Moreover, the dose recommendation in the current study was based on the $90 \%$ of virtually simulated patients. Physicians should be cautious of the risk of toxicity after taking a rescue dose in cases of poor compliance, especially in slow metabolizer, pediatric and elderly patients.

Our dose recommendations were based on selected dosing regimens with dosing intervals from 8-8-8 $\mathrm{h}$ to $4-10-10 \mathrm{~h}$ in TID regimens and from $10-14 \mathrm{~h}$ to $14-10 \mathrm{~h}$ in BID regimens. For other dosing regimens, further investigation may be needed to precisely map the CBZ and CBZE concentration-time profile and make the appropriate recommendations.

Population pharmacokinetic parameters of CBZ were derived from CBZ conventional preparation. It was reported that a twice-daily CBZ extended-release preparation can maintain small fluctuations of peak-to-trough within a dosing interval and may result in good tolerability in epilepsy patients ${ }^{[34,35]}$. Because its population pharmacokinetics were not well established, further research may be needed.

Although the current study cannot simulate all clinical settings, the findings could be helpful for the actual management of epilepsy based on therapeutic range, especially in light of the substantial degree of noncompliance observed in these patients. Our study showed that the dosing recommendations for delayed or missed doses are time related and regimen dependent. The co-therapy and body weight of the patient have no significant impact on the dose recommendation in the event of poor compliance. Furthermore, a Monte Carlo simulation based on population pharmacokinetics is a useful tool to investigate the impact of poor compliance and provide the rational dose recommendations for clinicians to sufficiently maintain the appropriate trough-peak concentration.

\section{Acknowledgements}

This study was supported by the National Natural Science Foundation of China (№ 81072702 and № 81071116) and the major research and development project on innovative drugs, China Ministry of Science and Technology (2012ZX09303004001)

The authors thank PhD candidates Mr Stephen Charles CROUCH and Miss Yu-zheng WANG (Albany College of 
Pharmacy and Health Sciences, NY, USA) for their critical reading and reviewing of the manuscript. The authors are also grateful to the anonymous reviewer for his valuable comments.

\section{Author contribution}

Zheng JIAO and Yi WANG designed research; Jun-jie DING, Zheng JIAO, and Yun-jian ZHANG performed the research and wrote the paper.

\section{References}

1 Bertilsson L, Tomson T. Clinical pharmacokinetics and pharmacological effects of carbamazepine and carbamazepine-10,11epoxide. An update. Clin Pharmacokinet 1986; 3: 177-98.

2 Bertilsson L. Clinical pharmacokinetics of carbamazepine. Clin Pharmacokinet 1978; 3: 128-43.

3 Potter J, Donnelly A. Carbamazepine-10,11-epoxide in therapeutic drug monitoring. Ther Drug Monit 1998; 6: 652-7.

4 Garnett W. Antiepileptic drugs. In: Schumacher G, editor. Therapeutic drug monitoring. Connecticut: Appleton and Lange Norwalk; 1995. p351-6.

5 Winnicka R, Topacinski B, Szymczak W, Szymanska B. Carbamazepine poisoning: elimination kinetics and quantitative relationship with carbamazepine 10,11-epoxide. J Toxicol Clin Toxicol 2002; 40: 75965.

6 Spina E, Pisani F, Perucca E. Clinically significant pharmacokinetic drug interactions with carbamazepine. An Update. Clin Pharmocokinet 1996; 31: 198-214.

7 Patsalos P, Berry D, Bourgeois B. Antiepileptic drugs - best practice guidelines for therapeutic drug monitoring: a position paper by the subcommission on therapeutic drug monitoring, ILAE Commission on Therapeutic Strategies. Epilepsia 2008; 49: 1239-76.

8 Manjunath R, Davis K, Candrilli S, Ettinger A. Association of antiepileptic drug nonadherence with risk of seizures in adults with epilepsy. Epilepsy Behav 2009; 14: 372-8.

9 Rosenfeld W, Bramley T, Meyer K. Patient compliance with topiramate vs other antiepileptic drugs: A claims database analysis. Epilepsia 2004; 457: 238-9.

10 Stanaway L, Lambie D, Johnson R. Non-compliance with anticonvulsant therapy as a cause of seizures. N Z Med J 1985; 98 : 150-2.

11 Cramer J, Glassman M, Rienzi V. The relationship between poor medication compliance and seizures. Epilepsy Behav 2002; 3: 33842.

12 Davis K, Candrilli S, Edin H. Prevalence and cost of nonadherence with antiepileptic drugs in an adult managed care population. Epilepsia 2008; 49: 446-54.

13 Bonate P. A brief introduction to Monte Carlo simulation. Clin Pharmacokinet 2001; 1: 15-22.

14 Jiao Z, Shi X, Zhao Z, Zhong M. Population pharmacokinetic modeling of steady state clearance of carbamazepine and its epoxide metabolite from sparse routine clinical data. J Clin Pharm Ther 2004; 29 : 247-56.

15 Jiao Z, Zhong M, Shi X, Zhang J. HPLC simultaneous determination of carbamazepine, carbamazepine-10,11-epoxide, phenytoin in human serum. Chin J Pharm Anal 2002; 22: 445-9.

16 Zhang R, Qu Z, Yu L, Wu Y, Fan C. Comparison on bioavailability of domestic and imported carbamazepine tablets in normal volunteers. Chin J Pharm 1990; (12): 544-5.

17 Eadie M. Therapeutic drug monitoring-antiepileptic drugs. Br J Clin
Pharmacol 2001; 52 Suppl 1: 11S-20S.

18 Gram L, Flachs H, Wurtz-Jorgensen A, Parnas J, Andersen B. Sodium valproate, serum level and clinical effect in epilepsy: a controlled study. Epilepsia 1979; 20: 303-11.

19 Harden CL, Zisfein J, Atos-Radzion E C, Tuchman AJ. Combination valproate-carbamazepine therapy in partial epilepsies resistant to carbamazepine monotherapy. J Epilepsy 1993; 6: 91-4.

20 Deckers C, Czuczwar S, Hekster Y, Keyser A, Kubova H, Meinardi H, et al. Selection of antiepileptic drug polytherapy based on mechanisms of action: the evidence reviewed. Epilepsia 2000; 41: 1364-74.

21 Ahmad A, Garnett W. Carbamazepine extended-release capsules vs. oxcarbazepine: computer simulations of the effect of missed doses on drug plasma concentrations. Curr Med Res Opin 2005; 21: 1363-8.

22 Garnett W, Mclean A, Zhang Y, Clausen S, Tulloch S. Simulation of the effect of patient nonadherence on plasma concentrations of carbamazepine from twice-daily extended-release capsules. Curr Med Res Opin 2003; 19: 519-25.

23 Faught R, Weiner J, Guerin A, Cunnington M, Duh M. Impact of nonadherence to antiepileptic drugs on health care utilization and costs: findings from the RANSOM study. Epilepsia 2009; 50: 501-9.

24 Schmidt D, Haenel F. Therapeutic plasma levels of phenytoin, phenobarbital, and carbamazepine: individual variation in relation to seizure frequency and type. Neurology 1984; 34: 1252-5.

25 Woo E, Chan Y, Yu Y, Chan Y, Huang C. If a well-stabilized epileptic patient has a subtherapeutic antiepileptic drug level, should the dose be increased? A randomized prospective study. Epilepsia 1988; 29: 129-39.

26 Gannaway D, Mawer G. Serum phenytoin concentration and clinical response in patients with epilepsy. Br J Clin Pharmacol 1981; 12: 833-9.

27 Perucca E. Is there a role for therapeutic drug monitoring of new anticonvulsants? Clin Pharmacokinet 2000; 38: 191-204.

28 Ahmad A, Douglas B, Barr W, Reed R, Garnett W. The use of Monte Carlo simulations to study the effect of poor compliance on the steady state concentrations of valproic acid following administration of enteric-coated and extended release divalproex sodium formulations. Biopharm Drug Dispos 2005; 26: 417-25.

29 Dutta S, Reed R. Effect of delayed and/or missed enteric-coated divalproex doses on valproic acid concentrations: simulation and dose replacement recommendations for the clinician. J Clin Pharm Ther 2006; 31: 321-9.

30 Jiao Z, Zhong M, Shi X, Hu M, Zhang J. Population pharmacokinetics of carbamazepine in Chinese epilepsy patients. Ther Drug Monit 2003; 25: 279-86.

31 Hermida J, Tutor J. How suitable are currently used carbamazepine immunoassays for quantifying carbamazepine-10,11-epoxide in serum samples? Ther Drug Monit 2003; 25: 384-8.

32 Jiao Z, Zhao Z, Shi X, Zhong M. Comparison of HPLC and FPIA method in determination of carbamazepine in human serum. Chin J Clin Pharmacol 2004; 20: 291-4.

33 Shen S, Elin R, Soldin S. Characterization of cross reactivity by carbamazepine 10,11-epoxide with carbamazepine assays. Clin Biochem 2001; 34: 157-8.

34 Garnett W, Levy B, Mclean A, Zhang Y, Couch RA, Rudnic EM, et al. Pharmacokinetic evaluation of twice-daily extended-release carbamazepine (CBZ) and four-times-daily immediate-release CBZ in patients with epilepsy. Epilepsia 1998; 39: 274-9.

35 Hogan R, Garnett W, Thadani V, Group CS. Tolerability and effects on quality of life of twice-daily extended-release carbamazepine in adults with seizure disorders an open-label, to month continuation study. Clin Ther 2003; 25: 2586-96. 